\title{
Complications and outcomes following inguinal lymphadenectomy for malignant melanoma in an Asian population
}

In A Lee', Hyun Jeong Kim¹, Eunjin Kim', Jee Youn Lee², Juhan Lee', Jae Geun Lee', Choong-kun Lee ${ }^{3}$, Sang Joon Shin ${ }^{3}$, Kee Yang Chung ${ }^{4}$, Myoung Soo Kim ${ }^{1}$

'Department of Surgery, Yonsei University College of Medicine, Seoul;

${ }^{2}$ Department of Surgery, Kangbuk Samsung Hospital, Sungkyunkwan University School of Medicine, Seoul;

${ }^{3}$ Division of Medical Oncology, Department of Internal Medicine and ${ }^{4}$ Department of Dermatology, Yonsei University College of Medicine, Seoul, Korea

Purpose: Melanoma is a potentially fatal cutaneous malignancy and regional lymph node (LN) metastases are the most important predictors of mortality. This study aimed to analyze clinical features and risk factors of complications associated with inguinal LN dissection (LND) to establish treatment protocols.

Methods: This single-center retrospective study (2000 to 2018) consisted of patients who underwent inguinal area sentinel LN biopsy (SLNB) or LND due to malignant melanoma. Risk factors and outcomes were analyzed.

Results: One hundred patients underwent SLNB alone $(n=67$; patients with negative SLNB), complete LND (CLND) after positive SLNB $(n=19)$, or radical LND without SLNB $(n=14)$. Five-year overall survival and disease-free survival rates among these groups were $87.3 \%$, $57.4 \%$, and $61.9 \%$, and $59.0 \%, 22.7 \%$, and $28.1 \%$, respectively. The complication rate in the SLNB alone group was lower than the other groups (22.4\% vs. $47.4 \%$ and $35.7 \%$, respectively; $P=0.048)$. Seroma was the most common complication in the SLNB alone group (15.0\%); lymphedema was most common in the CLND after SLNB group (21.1\%). Multivariate analysis of risk factors for postoperative complications found the hazard ratio for body mass index $>28 \mathrm{~kg} / \mathrm{m}^{2}$ was 4.376 (95\% confidence interval $[\mathrm{Cl}], 1.243-15.401 ; P=0.022$ ). The hazard ratio for LND (including CLND after SLNB and radical LND without SLNB) was $3.263(95 \% \mathrm{Cl}, 1.248-8.529 ; \mathrm{P}=0.016)$.

Conclusion: Inguinal LND is a higher risk procedure compared to SLNB and other sites for postoperative complications, irrespective of meticulous surgical techniques. More studies are needed to establish treatment protocols (e.g., observation vs. CLND after a positive SLNB result) and the risks and benefits in Asian populations.

Keywords: Melanoma, Complication, Outcome, Sentinel lymph node biopsy, Lymph node dissection

Received: Oct 9, 2020 Revised: Nov 24, 2020 Accepted: Dec 7, 2020

Correspondence to: Jae Geun Lee

Department of Surgery, Yonsei University College of Medicine,

50-1 Yonsei-ro, Seodaemun-gu, Seoul 03722, Korea

Tel: +82-2-2228-2138, Fax: +82-2-313-8289

E-mail:drjg1@yuhs.ac

ORCID: In A Lee (https://orcid.org/0000-0003-2739-0750), Hyun Jeong Kim (https://orcid.org/0000-0002-6033-5094), Eunjin Kim (https://orcid.org/00000002-5438-0649), Jee Youn Lee (https://orcid.org/0000-0002-5222-3295), Juhan Lee (https://orcid.org/0000-0003-4910-2596), Jae Geun Lee (https://orcid. org/0000-0002-6722-0257), Choong-kun Lee (https://orcid.org/0000-0001-51515096), Sang Joon Shin (https://orcid.org/0000-0001-5350-7241), Kee Yang Chung (https://orcid.org/0000-0003-3257-0297), Myoung Soo Kim (https://orcid. org/0000-0002-8975-8381)

Copyright $@ 2020$ Korean Society of Surgical Oncology

This is an Open Access article distributed under the terms of the Creative Commons Attribution Non-Commercial License (http://creativecommons.org/licenses/by-nc/4.0) which permits unrestricted non-commercial use, distribution, and reproduction in any medium, provided the original work is properly cited.

\section{INTRODUCTION}

Melanoma is a potentially fatal cutaneous malignancy that continues to increase in incidence worldwide. In 2019, there were more than 96,000 new diagnoses and 7,000 deaths in the United States [1]. Prognosis varies widely. Disease stage and regional lymph node $(\mathrm{LN})$ metastasis are the most important predictors of mortality in patients with melanoma [2].

Since Morton et al. [3] reported the concept of using sentinel LN biopsy (SLNB) for malignant melanoma staging, SLNB has become an important diagnostic tool for determining whether the cancer has spread beyond the primary site to the LN. Until recently, complete LN dissection (CLND) was recommended for a positive SLNB. However, the role of CLND is becoming increasingly controversial due to complications associated with lymph node 
dissection (LND) and the limited survival benefit $[2,4-6]$. The Multicenter Selective Lymphadenectomy Trial (MSLT)-II found that immediate CLND results in increased rates of regional disease control, but has no melanoma-specific survival benefit in patients with melanoma and sentinel LN metastasis [6].

Postlewait et al. [7] found that outcomes after axillary dissection for melanoma appear better than those described after inguinal lymphadenectomy. They suggested that complications are of less concern when determining whether a patient should undergo axillary dissection or observation. In contrast, inguinal LND has a higher rate of complications than axillary dissection ( $71 \%$ vs. $47 \%$, respectively) [8], and inguinal LND should not be recommended routinely in patients with a positive SLNB in the era of MSLT-II [9].

The populations included in previous studies were patients with melanoma in Western countries [4-6]. No risk-benefit studies of inguinal CLND after a positive SLNB have been performed in Asian populations. In general, Asian patients have lower rates of surgical complications than Caucasian patients due to lower rates of diseases including obesity and diabetes [10]. Thus, this study aimed to reveal the complications and risk factors associated with inguinal LND in an Asian population.

\section{METHODS}

The Institutional Review Board of Yonsei University approved this study for retrospective chart review and data collection (IRB No. 4-2020-0731). We reviewed the data of patients who underwent inguinal area SLNB or LND due to malignant melanoma at the Severance Hospital, Yonsei University College of Medicine, Seoul, Korea, between January 2000 and September 2018. The study population was divided into three groups: (1) SLNB alone (patients with negative SLNB results); (2) CLND after SLNB (patients who underwent immediate CLND after a positive SLNB result); and (3) radical LND (RLND) without SLNB (patients with confirmed inguinal LN metastasis based on needle aspiration biopsy or two more image modalities such as a computed tomography scan, ultrasound, or positron emission tomography-computed tomography scan).

SLNB was performed in patients diagnosed with malignant skin lesions. CLND was almost always performed in patients with an SLNB positive result. The primary tumor was excised by a dermatologist using wide excision or slow Mohs microscopic surgery. Inguinal area SLNB or LND was performed by a transplant surgeon using a $2.5 \times$ magnified Loupe to reduce the risk of seroma and lymphocele formation. All surgeries were performed at Severance Hospital.

On the day of SLNB, $18.5 \mathrm{Mbq}(0.5 \mathrm{mCi}) 99$ mTc-phytate (Korea
Atomic Energy Research Institute, Daejeon, Korea) diluted in 0.5 $\mathrm{mL}$ saline was injected at four points around the primary tumor. A lymphoscintigram was obtained to identify all basins at risk and the sentinel LN. In the operating room, a hand-held gamma detector (Navigator GPS, RMD Inc., Watertown, MA, USA) was used to identify sentinel LNs [11]. After the sentinel LN was harvested and measured again for radioactivity, the probe was used to search the harvested area to ensure there were no residual nodes with significant radioactivity. After complete removal of the primary melanoma and LN metastasis, adjuvant treatments were administered to patients with stage II or greater melanoma.

We reviewed the pathologic data based on the 8th edition of the American Joint Committee on Cancer staging system guidelines [12]. We also reviewed demographic, clinical, and oncologic outcome data. The statistical analysis was performed using SPSS 25 software (IBM Corp., Armonk, NY, USA). Results were expressed as mean \pm standard deviation values for continuous variables and as numbers (proportions) for categorical variables. Categorical variables were examined using Fisher exact tests or chi-square tests for between-group comparisons. Continuous variables were examined using independent Student t-tests.

Correlations between complications and risk factors were assessed using univariate and multivariate analysis. Factors that were significant $(\mathrm{P}<0.05)$ in the univariate analysis were included in a multivariate logistic regression model. Kaplan-Meier survival analysis was used to analyze survival rates according to each variable. Log-rank tests were used to compare survival rates between each group. P-values $<0.05$ were considered statistically significant.

\section{RESULTS}

\section{Patient population}

A total of 100 patients underwent SLNB alone ( $\mathrm{n}=67)$, CLND after SLNB $(n=19)$, or RLND without SLNB $(n=14)$. The mean age was $59.0 \pm 13.5$ years and the mean body mass index (BMI) was $24.6 \pm 3.2$ among all patients. The percentages of patients with hypertension, diabetes mellitus (DM), and of patients who smoked were $39 \%, 18 \%$, and $25 \%$, respectively. The proportion of patients with DM in the CLND after SLNB group (5.3\%) was slightly lower than the other groups. Most patients were American Society of Anesthesiologist class 2 or lower. There were no significant differences in demographic and clinical characteristics among the three groups. The results for clinical characteristics and comorbidities of the three groups are presented in Table 1.

\section{TNM stage and oncologic outcomes}

Comparison of TNM stages among the three groups revealed 
Table 1. Demographic and clinical data in patients who underwent inguinal area SLNB or LND, by melanoma treatment group

\begin{tabular}{lcccc}
\hline Variable & SLNB alone $(\mathrm{n}=67)$ & CLND after SLNB $(\mathrm{n}=19)$ & RLND without SLNB $(\mathrm{n}=14)$ & P-value \\
\hline Age $(\mathrm{yr})$ & $59.9 \pm 13.7$ & $55.9 \pm 14.1$ & $58.7 \pm 12.1$ & 0.524 \\
Height $(\mathrm{cm})$ & $161.4 \pm 7.9$ & $161.3 \pm 8.7$ & $163.6 \pm 9.9$ & 0.662 \\
Weight $(\mathrm{kg})$ & $65.2 \pm 9.3$ & $61.5 \pm 10.2$ & $64.0 \pm 12.0$ & 0.348 \\
BMI $\left(\mathrm{kg} / \mathrm{m}^{2}\right)$ & $25.0 \pm 3.1$ & $23.6 \pm 3.4$ & $23.9 \pm 3.2$ & 0.174 \\
HTN & $27(40.3)$ & $8(42.1)$ & $4(28.6)$ & 0.682 \\
DM & $13(19.4)$ & $1(5.3)$ & $4(28.6)$ & 0.198 \\
Smoking & $15(22.4)$ & $4(21.1)$ & $6(42.9)$ & 0.249 \\
ASA class & & & & 0.129 \\
1 & $21(31.3)$ & $9(47.4)$ & $9(64.3)$ & $5(35.7)$ \\
2 & $34(50.7)$ & $7(36.8)$ & 0 & \\
\hline
\end{tabular}

Values are presented as mean \pm standard deviation or number $(\%)$.

SLNB, sentinel lymph node biopsy; LND, lymph node dissection; CLND, complete LND; RLND, radical LND; BMI, body mass index; HTN, hypertension; DM, diabetes mellitus; ASA, American Society of Anesthesiologists.

Table 2. Pathologic T, N, and M stage according to AJCC 8th edition guidelines in patients who underwent inguinal area SLNB or LND, by melanoma treatment group

\begin{tabular}{lcccc}
\hline Stage & $\begin{array}{c}\text { SLNB alone } \\
(n=67)\end{array}$ & $\begin{array}{c}\text { CLND after } \\
\text { SLNB }(n=19)\end{array}$ & $\begin{array}{c}\text { RLND without } \\
\text { SLNB }(n=14)\end{array}$ & P-value \\
\hline T is & 0 & $1(5.3)$ & 0 & 0.050 \\
T 1a & $3(4.5)$ & 0 & 0 & \\
T 1b & $4(6.0)$ & 0 & $2(14.0)$ & \\
T 2a & $18(26.9)$ & $4(21.1)$ & $1(7.1)$ & \\
T 2b & $5(7.5)$ & $1(5.3)$ & 0 & \\
T 3a & $9(13.4)$ & $2(10.5)$ & $1(7.1)$ & \\
T 3b & $6(9.0)$ & $2(10.5)$ & $3(21.4)$ & \\
T 4a & $9(13.4)$ & $5(26.3)$ & 0 & \\
T 4b & $13(19.4)$ & $4(21.1)$ & $5(35.7)$ & \\
N 0 & $66(98.5)$ & 0 & $6(42.9)$ & $<0.001$ \\
N 1a & $1(1.5)$ & $15(78.9)$ & 0 & \\
N 1b & 0 & 0 & $3(21.4)$ & \\
N 2a & 0 & $3(15.8)$ & 0 & \\
N 3a & 0 & $1(5.3)$ & 0 & \\
N 3b & 0 & 0 & $1(7.1)$ & \\
N 3c & 0 & 0 & $2(14.3)$ & \\
M 0 & $67(100)$ & $18(94.7)$ & $12(100)$ & 0.122 \\
M 1 & 0 & $1(5.3)$ & 0 & \\
\hline
\end{tabular}

Values are presented as number (\%).

AJCC, American Joint Committee on Cancer; SLNB, sentinel lymph node biopsy; LND, lymph node dissection; CLND, complete LND; RLND, radical LND.

there were significant between-group differences in the $\mathrm{T}$ and $\mathrm{N}$ stages (Table 2). In the SLNB alone group, the greatest percentage was T stage 2 (34.4\%). T stage 4 was the highest percentage in the CLND after SLNB group and the RLND without SLNB group (47.4\% and $35.7 \%$, respectively) $(\mathrm{P}=0.05)$. For nodal stage, $\mathrm{N} 1$ was the highest in the CLND after SLNB group, with 1.5\% in the SLNB
Table 3. TNM stage according to AJCC 8th edition guidelines and oncologic outcome in patients who underwent inguinal area SLNB or LND, by melanoma treatment group

\begin{tabular}{lcccr}
\hline & $\begin{array}{c}\text { SLNB alone } \\
(\mathrm{n}=67)\end{array}$ & $\begin{array}{c}\text { CLND after } \\
\text { SLNB }(\mathrm{n}=19)\end{array}$ & $\begin{array}{c}\text { RLND without } \\
\text { SLNB }(\mathrm{n}=14)\end{array}$ & P-value \\
\hline $\begin{array}{l}\text { TNM stage } \\
\text { Stage I }\end{array}$ & $25(37.3)$ & 0 & $2(16.7)$ & $<0.001$ \\
Stage II & $41(61.2)$ & 0 & $4(33.3)$ & \\
Stage III & $1(1.5)$ & $17(89.5)$ & $6(50.0)$ & \\
Stage IV & 0 & $1(5.3)$ & 0 & \\
Adjuvant RTx & $5(7.5)$ & $8(42.1)$ & $9(64.3)$ & 0.012 \\
Adjuvant CTx & $26(38.8)$ & $17(89.5)$ & $13(92.9)$ & $<0.001$ \\
Recurrence & $22(32.8)$ & $13(68.4)$ & $9(64.3)$ & 0.006 \\
Death & $5(7.5)$ & $6(31.6)$ & $5(35.7)$ & 0.004 \\
\hline
\end{tabular}

Values are presented as number (\%).

AJCC, American Joint Committee on Cancer; SLNB, sentinel lymph node biopsy; LND, lymph node dissection; CLND, complete LND; RLND, radical LND; RTX, radiation; $\mathrm{CT}$, chemotherapy.

alone group, $78.9 \%$ in the CLND after SLNB group, and $21.4 \%$ in the RLND without SLNB group. The CLND after SLNB group had 15.8\% N2 tumors; the remaining groups had 0\% classified as N2 tumors. The RLND without SLNB group had the greatest proportion of $\mathrm{N} 3$ tumors (21.4\%); there were $0 \%$ in the SLNB alone group, and $5.3 \%$ in the CLND after SLNB group $(\mathrm{P}<0.001)$. The only case of stage M1 was in the CLND after SLNB group, which was diagnosed using additional imaging after surgery; all other groups had stage $\mathrm{M} 0$ tumors only $(\mathrm{P}=0.122)$.

Comparison of each group by TNM stage revealed that the SLNB alone group had $37.3 \%$ in stage I and $61.2 \%$ in stage II (Table 3 ). The SLNB alone group had more early-stage tumors than the other two groups. A total of $89.5 \%$ of the tumors in the CLND after 
SLNB group and 50\% of the tumors in the RLND without SLNB group were stage III. The CLND after SLNB group and the RLND without SLNB group had significantly greater proportions of advanced-stage tumors $(\mathrm{P}<0.001)$. The results for additional adjuvant therapy after surgery indicated that both adjuvant radiotherapy and chemotherapy were performed in greater proportions of the cases in the RLND without SLNB group, followed by the CLND after SLNB group and the SLNB alone group (radiotherapy, $\mathrm{P}=0.012$; chemotherapy, $\mathrm{P}<0.001$ ) (Table 3).

The analysis for 3 years and 5 years of overall survival in the SLNB, CLND after SLNB, and RLND without SLNB groups found $91.2 \%, 71.8 \%$, and $62.3 \%$ survived for 3 years, respectively, and $87.3 \%, 57.4 \%$, and $61.9 \%$ survived for 5 years, respectively. The proportions with disease-free survival in the treatment groups were $63.5 \%, 30.2 \%$, and $37.5 \%$, respectively, for 3 years, and $59.0 \%$, $22.7 \%$, and $28.1 \%$, respectively, for 5 years.

Compared with the patients in the CLND after SLNB group and the RLND without SLNB group, the patients in the SLNB alone

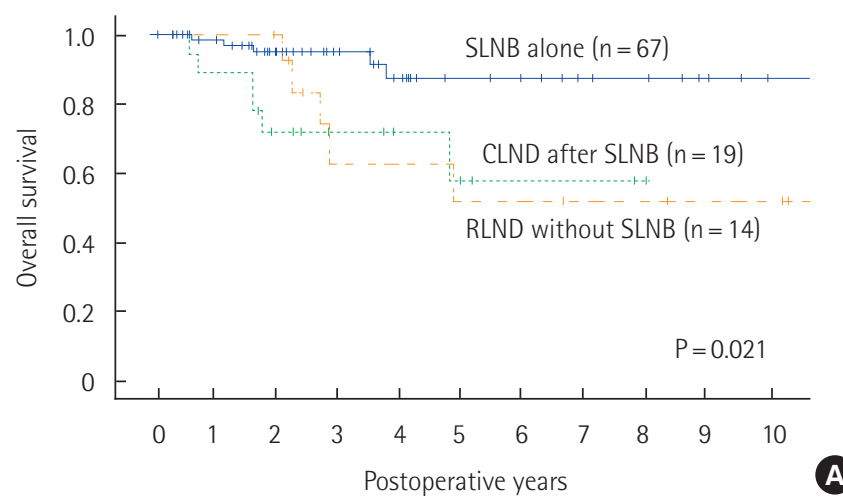

group had better overall survival $(\mathrm{P}=0.010$ and $\mathrm{P}=0.027$, respectively) and disease-free survival $(\mathrm{P}=0.002$ and $\mathrm{P}=0.071$, respectively) (Fig. 1). Disease-free survival and overall survival at each TNM stage were also significantly different between groups $(\mathrm{P}<0.001$ for both) (Fig. 2).

\section{Complications and risk factors}

We compared the incidence of postoperative complications according to width of the inguinal LND (Table 4). The SLNB alone group had three patients (4.5\%), the CLND after SLNB group had 16 patients (84.2\%), and the RLND without SLNB group had 10 patients $(71.4 \%)$, who required indwelling drain insertion $(\mathrm{P}<0.001)$. The mean values for duration of indwelling drains in the SLNB alone, the CLND after SLNB, and the RND without SLNB groups were $7.5 \pm 9.2,17.3 \pm 14.3$, and $27.4 \pm 22.1$ days, respectively. Hospital stay lengths after each surgery were $7.5 \pm 5.6$ days for the SLNB alone group, 11.8 \pm 2.7 days for the CLND after SLNB group, and $16.6 \pm 4.4$ days for the RLND without SLNB

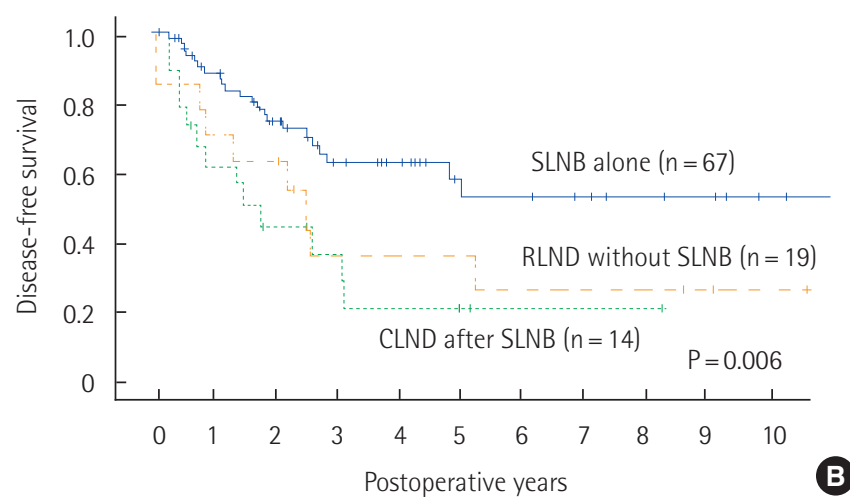

Fig. 1. Overall (A) and disease-free survival (B) according to type of surgery performed in inguinal area, by melanoma treatment group. (A) Overall survival; SLNB alone vs. CLND after SLNB $(P=0.010)$ and SLNB alone vs. RLND without SLNB $(P=0.027)$. (B) Disease-free survival; SLNB alone vs. CLND after SLNB $(P=0.002)$ and SLNB alone vs. RLND without SLNB $(P=0.071)$. SLNB, sentinel lymph node biopsy; LND, lymph node dissection; CLND, complete LND; RLND, radical LND.
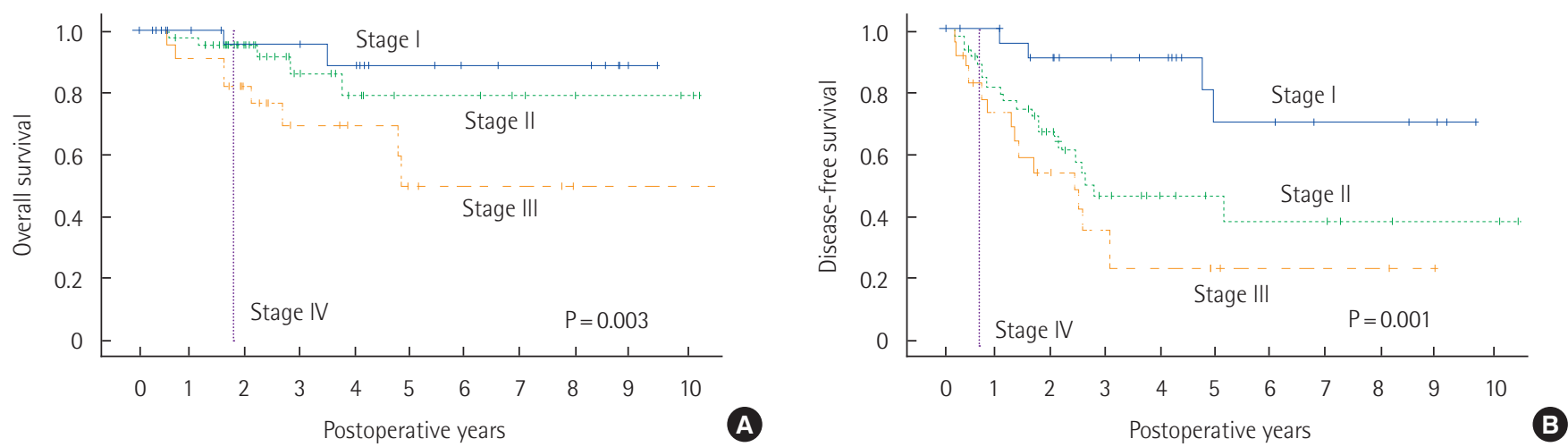

Fig. 2. Overall (A) and disease-free survival (B) according to TNM stage in patients who underwent inguinal area sentinel lymph node biopsy or lymph node dissection, by melanoma treatment group. 
Table 4. Complications and hospital stay characteristics in patients who underwent inguinal area SLNB or LND, by melanoma treatment group

\begin{tabular}{lcccr}
\hline Characteristics & SLNB alone $(n=67)$ & CLND after SLNB $(n=19)$ & RLND without SLNB $(n=14)$ & P-value \\
\hline Drain indwelling & $3(4.5)$ & $16(84.2)$ & $10(71.4)$ & $<0.001$ \\
Duration of indwelling (day) & $7.5 \pm 9.2$ & $17.3 \pm 14.3$ & $27.4 \pm 22.1$ & 0.219 \\
Hospital stay (day) & $7.5 \pm 5.6$ & $11.8 \pm 2.7$ & $5(35.7)$ & $<.001$ \\
Complication & $15(22.4)$ & $9(47.4)$ & $2(14.3)$ & 0.048 \\
Complication type & & & $2(14.3)$ \\
Seroma & $10(15.0)$ & $3(15.8)$ & 0 & 0.022 \\
Lymphedema & 0 & $4(21.1)$ & $1(7.1)$ & 0 \\
Wound infection & $3(4.5)$ & $2(10.5)$ & 0 & 0 \\
Wound necrosis & 0 & 0 & \\
Wound dehiscence & $2(3.0)$ & 0 & \\
\hline
\end{tabular}

Values are presented as number (\%) or mean \pm standard deviation.

SLNB, sentinel lymph node biopsy; LND, lymph node dissection; CLND, complete LND; RLND, radical LND.

Table 5. Univariate analysis of risk factor for complications in patients who underwent inguinal area SLNB or LND, by melanoma treatment group

\begin{tabular}{lccc}
\hline Factor & $\begin{array}{c}\text { Complication } \\
(\mathrm{n}=27)\end{array}$ & $\begin{array}{c}\text { No complication } \\
(\mathrm{n}=71)\end{array}$ & P-value \\
\hline Male sex & $12(44.4)$ & $31(43.7)$ & 1.000 \\
Age $>65 \mathrm{yr}$ & $7(25.9)$ & $22(31.0)$ & 0.805 \\
BMI $>28 \mathrm{~kg} / \mathrm{m}^{2}$ & $7(25.9)$ & $6(8.5)$ & 0.041 \\
Smoking & $9(33.3)$ & $16(22.5)$ & 0.305 \\
HTN & $11(40.7)$ & $27(38.0)$ & 0.820 \\
DM & $4(14.8)$ & $14(19.7)$ & 0.772 \\
Inguinal LND & $14(51.9)$ & $19(26.8)$ & 0.030 \\
Adjuvant CTx & $20(74.1)$ & $35(49.3)$ & 0.040 \\
Adjuvant RTx & $8(29.6)$ & $10(14.1)$ & 0.087 \\
ASA class & & & 0.121 \\
1 & $11(40.7)$ & $28(39.4)$ & \\
2 & $15(55.6)$ & $29(40.8)$ & \\
3 & $1(3.7)$ & $14(19.7)$ & \\
\hline
\end{tabular}

Values are presented as number (\%).

SLNB, sentinel lymph node biopsy; LND, lymph node dissection; BMI, body mass index; HTN, hypertension; DM, diabetes mellitus; CTx, chemotherapy; $R T x$, radiation; $A S A$, American Society of Anesthesiologists.

group $(\mathrm{P}<0.001)$.

Complications after surgery occurred in $22.4 \%$ of the SLNB alone, $47.4 \%$ of the CLND after SLNB, and 35.7\% of the RLND without SLNB, group patients (Table 4). The SLNB alone group had relatively fewer complications than the other two groups. The postoperative complications were wound seroma (15.0\%), lymphedema (6.0\%), wound infection (5.0\%), wound necrosis (1.0\%), and wound dehiscence (2.0\%). Seroma was the most common complication in the SLNB alone group (15.0\%) and lymphedema was the most common complication in the CLND after SLNB group (21.1\%). Seroma and lymphedema were equally common in the RLND without SLNB group (14.3\% for both) (Table 4).

The univariate analysis of risk factors for complications after surgery in patients receiving inguinal SLNB or dissection revealed that $\mathrm{BMI}>28 \mathrm{~kg} / \mathrm{m}^{2}(\mathrm{P}=0.041)$, inguinal LND (including CLND after SLNB and RLND without SLNB; $\mathrm{P}=0.030$ ), and adjuvant chemotherapy $(\mathrm{P}=0.040)$ were significantly associated with postoperative complications (Table 5).

The multivariate analysis of risk factors for postoperative complications in the inguinal area for melanoma found that for BMI $>28 \mathrm{~kg} / \mathrm{m}^{2}$, the hazard ratio was 4.376 (95\% confidence interval [CI], 1.243-15.401; $\mathrm{P}=0.022)$. For LND compared to SLNB, the hazard ratio was $3.263(95 \% \mathrm{CI}, 1.248-8.529 ; \mathrm{P}=0.016)$.

\section{DISCUSSION}

This study found complication rates and risk factors for inguinal area SLNB or LND in patients with melanoma in an Asian population. Malignant melanoma is one of the most rapidly increasing cancers worldwide [13]. The age-standardized mortality rates for malignant melanoma in Asian populations are relatively low, ranging from 0.18 to 0.23 [14]. Melanoma also generally has lower rates of complications in Asian patients than Western patients because of lower rates of diseases including obesity and diabetes [10]. However, despite research findings regarding these characteristics of Asian populations, the data for decision-making and prediction of complications in inguinal LND in terms of observation versus immediate CLND after an SLNB positive result are relatively scarce.

This study revealed complication rates of $22.4 \%$ after SLNB alone and of $47.4 \%$ for CLND after SLNB. These results are similar to those of a previous study. Morton et al. [15] found complication rates of $8.1 \%$ after SLNB alone and of 37.2\% for CLND after SLNB 
Table 6. Published literature reporting postoperative complications following inguinal area LND for melanoma treatment

\begin{tabular}{|c|c|c|c|c|c|c|c|}
\hline Author & Year & No. of patients & Study design & Infection (\%) & Seroma (\%) & $\begin{array}{l}\text { Dehiscence/ } \\
\text { necrosis (\%) }\end{array}$ & Lymphedema (\%) \\
\hline Shaw [19] & 1990 & 58 & Retrospective & 25 & 12 & 10 & 23 \\
\hline Coit [20] & 1991 & 42 & Prospective & $19 / 45^{a)}$ & - & - & - \\
\hline Beitsch [21] & 1992 & 168 & Retrospective & 29 & 14 & 26 & 44 \\
\hline Karakousis [22] & 1994 & 205 & Retrospective & 16 & 5 & 8 & 40 \\
\hline Hughes [23] & 2000 & 132 & Retrospective & 15 & 2 & 7 & 36 \\
\hline Serpell [8] & 2003 & 28 & Prospective & 25 & 46 & 25 & 29 \\
\hline de Vries [24] & 2006 & 14 & Retrospective & 29 & 7 & 7 & 64 \\
\hline Sabel [25] & 2007 & 212 & Retrospective & 19 & - & - & 27 \\
\hline Guggenheim [26] & 2008 & 43 & Retrospective & 33 & 42 & 7 & - \\
\hline Chang [18] & 2010 & 53 & Prospective & 55 & 28 & 53 & 45 \\
\hline Glarner [27] & 2013 & 281 & Retrospective & 13 & - & 2 & - \\
\hline \multirow[t]{2}{*}{ Bertheuil [28] } & 2015 & $27(V)$ & Retrospective & 11 & 30 & 15 & 37 \\
\hline & & $26(T)$ & & 0 & 23 & 19 & 27 \\
\hline Faut [29] & 2017 & 240 & Retrospective & 30 & 22 & 14 & - \\
\hline \multirow[t]{2}{*}{ Jorgensen [17] } & 2019 & $41(C)$ & Retrospective & 66 & 90 & - & 36 \\
\hline & & 14 (NP) & & 43 & 29 & - & 51 \\
\hline Current study $y^{b)}$ & 2020 & 33 & Retrospective & 6 & 15 & 3 & 18 \\
\hline
\end{tabular}

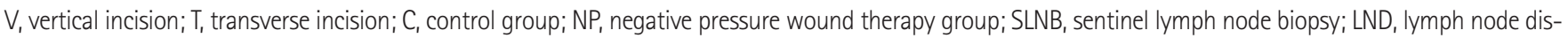
section; CLND, complete LND; RLND, radical LND.

${ }^{a)}$ Major infection/minor infection. ${ }^{b)}$ LND including CLND after SLNB and RLND without SLNB.

in MSLT-I patients. However, the MSLT-I had only a small proportion of inguinal area tumors because it also included axillary, neck, and inguinal dissection; $42 \%$ of the patients had a primary tumor in the upper or lower extremities, or both. LND in the inguinal area is higher risk than the axillary and neck areas because lymphatic vessel transection causing accumulation of lymphatic fluid in the dead space during inguinal LND is unavoidable [16]. These seromas and lymphoceles can lead to postoperative wound infection, skin necrosis, and wound dehiscence. Jorgensen et al. [17] found that the risk of lymphedema is significantly greater in the inguinal area than the axillary area (hazard ratio [HR], 2.49; $95 \% \mathrm{CI}, 1.34-4.55 ; \mathrm{P}<0.05)$. The rate of seroma formation is also greater for inguinal CLND than for axillary CLND.

Chang et al.'s prospective cohort study of patients with melanoma found that $77 \%$ of patients undergoing inguinal area LND experience complications, including infection (55\%), wound dehiscence (53\%), and lymphedema (85\%) [18]. High rates of infection (15\%-33\%), seroma (2\%-90\%), dehiscence/necrosis $(2 \%-53 \%)$, and lymphedema $(23 \%-64 \%)$ have also been found in other studies (Table 6) [8,17-29]. In this study, the complication rate was lower than in previous studies of postoperative complications following inguinal LND for melanoma. This difference may have resulted from the retrospective study design. We found very low rates of wound necrosis and dehiscence (only one patient [3.0\%] among
14 patients with RLND without SLNB and 19 patients with CLND after without SLNB).

To prevent lymphatic leaks during malignant melanoma surgery in the inguinal area, the transplant surgeon meticulously ligated lymphatic vessels using 3-0 or 4-0 silk and a Loupe with $2.5 \times$ magnification. After dissection, the operator sutured the subcutaneous layer using thick layers and the skin with minimal tension to avoid wound necrosis and dehiscence. We typically inserted an indwelling drain (84.2\% of patients with CLND after SLNB) and removed the drain several weeks post-surgery. We did not use prophylactic incisional negative wound therapy for inguinal LND in patients with melanoma. Study results suggest that use of incisional negative wound therapy can prevent wound complications, especially seroma and lymphocele [17]. However, randomized control design studies of oncologic outcomes and cost-effectiveness of this approach are scarce.

Lymphedema was found in $21.1 \%$ of CLND after SLNB patients and $14.3 \%$ of RLND without SLNB patients. In the MSLT-II, lymphedema was found in $24.1 \%$ of patients in CLND and $6.3 \%$ of those in the SLNB alone group [6]. Many incisional surgical approaches (e.g., vertical and transverse incision) to reduce lymphedema were attempted at our center. However, consistent with the results of a previous study, there was no one specific approach (i.e., a gold standard) that was suitable for all patients treated at our cen- 
ter [28].

Compared with SLNB, inguinal LND was a procedure associated with a high risk of complications after inguinal surgery in patients with malignant melanoma (HR, 3.263; 95\% CI, 1.248-8.529; $\mathrm{P}=0.016$ ). Similar to previous study results, we found that obesity $\left(\right.$ BMI $\left.>28 \mathrm{~kg} / \mathrm{m}^{2}\right)$ was an independent risk factor for complications. Diabetes and old age are also known risk factors for morbidity $[8,18,25,27]$. However, our univariate and multivariate analyses did not find significant associations between these factors and the risk of complications.

For regional node control, the 3-year disease-free survival was slightly higher in the CLND group than in the sentinel LN positive observation group $(68 \% \pm 1.7 \%$ vs. $63 \% \pm 1.7 \%$, respectively); the MSLT-II had a higher rate of regional disease control at 3 years $(92 \% \pm 1.0 \%$ vs. $77 \% \pm 1.5 \%$, respectively, $\mathrm{P}<0.001)$ [6]. The MSLTII found that non-sentinel LN metastases were present in $11.5 \%$ of patients in the dissection group; two of 19 patients (10.5\%) in the CLND after SLNB positive group had non-sentinel LN in this study. This study had no patients with observations after they were sentinel LN positive because the study period was before the MSLT-II. It is not possible to compare immediate CLND with observation after SLNB positivity. Nevertheless, CLND with SLNB positivity might also have had the benefit of regional disease control in this study because the rate of non-sentinel LN metastasis in patients with CLND was similar to that of the MSLT-II.

This study found survival differences according to TNM stage and overall survival and disease-free survival for patients in the SLNB alone group. Patients with negative SLNB had greater survival than those with CLND after SLNB positive or RND without SLNB. Because of the treatment policy before the MSLT-II, this result could be associated with the TNM stage difference between the SLNB alone group and the other groups. More studies are needed to compare survival benefits after SLNB alone and CLND after SLNB in Asian populations in the MSLT-II era.

This study had some limitations. The retrospective, single-center study design has limitations in revealing overall or disease-free survival benefits. There were no patients with observations after a sentinel LN positive result because the study period was before the MSLT-II. It was not possible to compare immediate CLND with observations after an SLNB positive result. Nevertheless, this study is the first to report rates and risk factors for complications associated with inguinal area SLNB and LND for melanoma.

In conclusion, compared with other sites and for those other than SLNB, inguinal LND is a procedure associated with relatively high risks for postoperative complications, even when meticulous surgical technique is used. Treatment of patients with obesity (BMI $>28 \mathrm{~kg} / \mathrm{m}^{2}$ ) and other risk factors for complications should be considered carefully. More studies are needed to establish treatment protocols (e.g., observation vs. CLND after a positive SLNB result) and the risks and benefits in Asian populations.

\section{CONFLICT OF INTEREST}

No potential conflict of interest relevant to this article was reported.

\section{REFERENCES}

1. Enomoto LM, Levine EA, Shen P, Votanopoulos KI. Role of surgery for metastatic melanoma. Surg Clin North Am 2020;100:12739.

2. Balch CM, Gershenwald JE, Soong SJ, Thompson JF, Atkins MB, Byrd DR, et al. Final version of 2009 AJCC melanoma staging and classification. JClin Oncol 2009;27:6199-206.

3. Morton DL, Wen DR, Wong JH, Economou JS, Cagle LA, Storm FK, et al. Technical details of intraoperative lymphatic mapping for early stage melanoma. Arch Surg 1992;127:392-9.

4. Morton DL, Thompson JF, Cochran AJ, Mozzillo N, Elashoff R, Essner R, et al. Sentinel-node biopsy or nodal observation in melanoma. N Engl J Med 2006;355:1307-17.

5. Morton DL, Thompson JF, Cochran AJ, Mozzillo N, Nieweg OE, Roses DF, et al. Final trial report of sentinel-node biopsy versus nodal observation in melanoma. N Engl J Med 2014;370:599-609.

6. Faries MB, Thompson JF, Cochran AJ, Andtbacka RH, Mozzillo N, Zager JS, et al. Completion dissection or observation for sentinel-node metastasis in melanoma. N Engl J Med 2017;376:221122.

7. Postlewait LM, Farley CR, Seamens AM, Le N, Rizzo M, Russell MC, et al. Morbidity and outcomes following axillary lymphadenectomy for melanoma: weighing the risk of surgery in the era of MSLT-II. Ann Surg Oncol 2018;25:465-70.

8. Serpell JW, Carne PW, Bailey M. Radical lymph node dissection for melanoma. ANZ J Surg 2003;73:294-9.

9. Peach H, Board R, Cook M, Corrie P, Ellis S, Geh J, et al. Current role of sentinel lymph node biopsy in the management of cutaneous melanoma: a UK consensus statement. J Plast Reconstr Aesthet Surg 2020;73:36-42.

10. Haider AH, Scott VK, Rehman KA, Velopulos C, Bentley JM, Cornwell EE 3rd, et al. Racial disparities in surgical care and outcomes in the United States: a comprehensive review of patient, provider, and systemic factors. J Am Coll Surg 2013;216:482-92.

11. Han HJ, Kim JR, Nam HR, Keum KC, Suh CO, Kim YB. Clinical outcomes after sentinel lymph node biopsy in clinically node-negative breast cancer patients. Radiat Oncol J 2014;32:132-7. 
12. Gershenwald JE, Scolyer RA. Melanoma staging: American Joint Committee on Cancer (AJCC) 8th edition and beyond. Ann Surg Oncol 2018;25:2105-10.

13. Nikolaou V, Stratigos AJ. Emerging trends in the epidemiology of melanoma. Br J Dermatol 2014;170:11-9.

14. Chen L, Jin S. Trends in mortality rates of cutaneous melanoma in East Asian populations. PeerJ 2016;4:e2809.

15. Morton DL, Cochran AJ, Thompson JF, Elashoff R, Essner R, Glass EC, et al. Sentinel node biopsy for early-stage melanoma: accuracy and morbidity in MSLT-I, an international multicenter trial. Ann Surg 2005;242:302-11.

16. Gerken ALH, Dobroschke J, Reissfelder C, Hetjens S, Braun V, Di Monta G, et al. Tissue sealants for the prevention of lymphoceles after radical inguinal lymph node dissection in patients with melanoma: a systematic review and individual patient data meta-analysis. J Surg Oncol 2019;119:728-36.

17. Jorgensen MG, Toyserkani NM, Thomsen JB, Sorensen JA. Prophylactic incisional negative pressure wound therapy shows promising results in prevention of wound complications following inguinal lymph node dissection for melanoma: a retrospective case-control series. J Plast Reconstr Aesthet Surg 2019;72:1178-83.

18. Chang SB, Askew RL, Xing Y, Weaver S, Gershenwald JE, Lee JE, et al. Prospective assessment of postoperative complications and associated costs following inguinal lymph node dissection (ILND) in melanoma patients. Ann Surg Oncol 2010;17:2764-72.

19. Shaw JH, Rumball EM. Complications and local recurrence following lymphadenectomy. Br J Surg 1990;77:760-4.

20. Coit DG, Peters M, Brennan MF. A prospective randomized trial of perioperative cefazolin treatment in axillary and groin dissection. Arch Surg 1991;126:1366-71.
21. Beitsch P, Balch C. Operative morbidity and risk factor assessment in melanoma patients undergoing inguinal lymph node dissection. Am J Surg 1992;164:462-5.

22. Karakousis CP, Driscoll DL. Groin dissection in malignant melanoma. Br J Surg 1994;81:1771-4.

23. Hughes TM, A'Hern RP, Thomas JM. Prognosis and surgical management of patients with palpable inguinal lymph node metastases from melanoma. Br J Surg 2000;87:892-901.

24. de Vries M, Vonkeman WG, van Ginkel RJ, Hoekstra HJ. Morbidity after inguinal sentinel lymph node biopsy and completion lymph node dissection in patients with cutaneous melanoma. Eur J Surg Oncol 2006;32:785-9.

25. Sabel MS, Griffith KA, Arora A, Shargorodsky J, Blazer DG 3rd, Rees $\mathrm{R}$, et al. Inguinal node dissection for melanoma in the era of sentinel lymph node biopsy. Surgery 2007;141:728-35.

26. Guggenheim MM, Hug U, Jung FJ, Rousson V, Aust MC, Calcagni $\mathrm{M}$, et al. Morbidity and recurrence after completion lymph node dissection following sentinel lymph node biopsy in cutaneous malignant melanoma. Ann Surg 2008;247:687-93.

27. Glarner CE, Greenblatt DY, Rettammel RJ, Neuman HB, Weber $\mathrm{SM}$. Wound complications after inguinal lymph node dissection for melanoma: is ACS NSQIP adequate? Ann Surg Oncol 2013; 20:2049-55.

28. Bertheuil N, Sulpice L, Levi Sandri GB, Lavoue V, Watier E, Meunier B. Inguinal lymphadenectomy for stage III melanoma: a comparative study of two surgical approaches at the onset of lymphoedema. Eur J Surg Oncol 2015;41:215-9.

29. Faut M, Heidema RM, Hoekstra HJ, van Ginkel RJ, Been SL, Kruijff S, et al. Morbidity after inguinal lymph node dissections: it is time for a change. Ann Surg Oncol 2017;24:330-9. 